\title{
Inondations des villes, inondations des champs
}

\section{Norme et territoire dans la prévention des inondations sur l'île de la Barthelasse (Avignon)}

\author{
Jessica Gentric ${ }^{1}$, Julien Langumier ${ }^{2}$ \\ 1 Urbaniste, DDE de la Manche, BP 496, boulevard de la Dollée, 50006 Saint-Lô cedex, France \\ 2 Ethnologue, ENTPE, UMR5600 RIVES, Rue Maurice Audin, 69120 Vaulx-en-Velin, France
}

L'approche des risques naturels connaît aujourd'hui de sensibles évolutions. Les politiques de prévention, les dispositifs de gestion de crise ne sont plus considérés comme de simples applications de techniques, normes et plans mais comme les produits de multiples compromis entre divers acteurs. De même, les personnes, groupes sociaux et collectivités directement en prise avec les risques n'apparaissent plus comme de simples cibles de ces politiques mais comme des acteurs à prendre en compte en tant que tels. Ces changements qui semblent a priori correspondre à un plus grand pragmatisme, à une plus grande reconnaissance des «parties prenantes » ne vont cependant pas sans ambigüités. Le double regard d'une urbaniste et d'un ethnologue porté sur un cas spécifique a ainsi permis de mettre en évidence la diversité des dynamiques résultant des tensions persistantes entre norme et territoire.

La Rédaction

\section{Mots-clés : \\ inondations ; \\ risque; \\ pluridisciplinarité ; ethnographie ; \\ île de la Barthelasse (Avignon)}

\section{Keywords: \\ floods; \\ Barthelasse Island; \\ Avignon; \\ river Rhone; \\ multidisciplinarity; \\ ethnography}

Résumé - À partir d'une enquête de terrain conduite sur l'île de la Barthelasse, l'article aborde la question de la prévention des inondations à travers le prisme de la tension entre norme et territoire. Le maintien de l'inondabilité de cette île agricole a toujours contribué à la protection du centre-ville d'Avignon. La démarche ethnographique traduit alors la manière dont se construit, dans les aménagements contre les crues, une solidarité entre Barthelassiens et Avignonnais. Elle tente également de saisir les pratiques locales qui se développent dans l'ombre des politiques publiques. En particulier, la tension entre norme et territoire est déclinée selon le clivage entre la ville et la campagne, entre les gestionnaires locaux et les aménageurs de la Compagnie nationale du Rhône, entre les habitants d'un territoire qui vivent avec les crues et les porteurs des politiques de prévention qui appellent au développement d'une «culture du risque ».

\begin{abstract}
Rural floods, urban floods. General standards and local situations in flood prevention on Barthelasse Island. Based on an ethnographical approach set on Barthelasse, an island on the river Rhone across from Avignon, our paper analyses how the issue of flooding is at the root of tensions between local interests and general policies. The island is still an agricultural area despite the influence of Avignon urban growth. Barthelasse Island has always been maintained as a floodable area to protect the low-lying city of Avignon. Such a context highlights the complexity of the relations, consisting simultaneously of solidarity and conflict, between the island farmers and the urban inhabitants of Avignon. Moreover, the river Rhone was altered in the 1970's to produce hydroelectricity. Nowadays it is no longer natural but artificial. Some areas like Barthelasse are still liable to flooding whereas others have got a better deal. The study investigates the relations between inhabitants who demand the building of new dykes and stakeholders who try to maintain a general plan for the area. Finally, the survey focuses on the people living in these risk prone areas and analyses the way they live with the risk. Confrontation between technical rationalities and the practices of inhabitants, the local and general scale of the issue and the former natural and present artificial state of the river question the idea of a "risk culture".
\end{abstract}

Auteur correspondant : langumier@yahoo.fr J. Langumier est chercheur associé au laboratoire RIVES et cofondateur de l'Association pour la recherche sur les catastrophes et les risques en anthropologie (ARCRA). 
Délimitée par le canal d'amenée à hauteur de Villeneuve-lès-Avignon et le "Vieux » Rhône en face d'Avignon, l'île de la Barthelasse s'étend sur sept cents hectares, dont quatre cents cultivables, et constitue la plus grande île fluviale de France (figure). Suivant les caprices du fleuve, certains îlots se sont rattachés à la rive gardoise, d'autres à la rive vauclusienne, tandis qu'une sorte d'archipel à géographie instable a formé, au cœur du lit mineur, l'île de la Barthelasse dont la partie sud conserve le nom d'île Piot. Cette situation «flottante » a été figée en 1971 par les aménagements de la Compagnie nationale du Rhône (CNR) dévolus à l'exploitation du potentiel hydroélectrique du fleuve. Deux barrages et une écluse ont été édifiés et le Rhône a été endigué sur toute sa longueur, sauf dans le Vieux Rhône passant à Avignon. Aujourd'hui, l'île de la Barthelasse, rattachée à la municipalité d'Avignon, compte environ mille habitants et se trouve régulièrement inondée par près de deux mètres d'eau, comme ce fut le cas en 1993, 1994 et, plus récemment, en 2002 et 2003.

Dans le contexte d'une territorialisation de la gestion des risques, $\mathrm{l}^{\prime}$ enquête monographique ${ }^{1}$ conduite à la Barthelasse révèle les tensions entre, d'une part, le « discours du code » (Barel, 1981) qui préside à la gestion de cet espace par une municipalité urbaine, à l'exploitation du fleuve par la CNR, ainsi qu'à la politique de prévention des risques, et, d'autre part, les pratiques locales développées par les habitants, sur cette île agricole, en matière de protection contre les crues ou de gestion des digues. Sur le plan méthodologique, l'association d'une urbaniste et d'un ethnologue permet de proposer un regard ethnographique sur un sujet d'aménagement, d'analyser la parole des populations sur une question traitée $\mathrm{d}^{\prime}$ habitude dans le champ de l'expertise, de faire enfin émerger les enjeux sociaux d'un problème environnemental ${ }^{2}$.

La ville d'Avignon bénéficie de l'inondation de la Barthelasse, car le volume d'eau qui s'épanche dans l'île fluviale déleste d'autant la pression du Rhône contre les remparts du centre-ville. Comment se joue alors la solidarité territoriale face aux inondations, dans un contexte marqué par le clivage entre ville et campagne, par les tensions entre Barthelassiens et Avignonnais?

L'histoire de la gestion du risque d'inondation sur l'île de la Barthelasse conduit aujourd'hui à la coexistence

\footnotetext{
1 Recherche conduite par Jessica Gentric (2007) et encadrée par Julien Langumier dans le cadre du master de l'Institut d'urbanisme de Lyon. L'enquête de terrain a permis la réalisation d'une vingtaine d'entretiens auprès des habitants et des responsables institutionnels (services de l'État déconcentrés, services municipaux, élus), la consultation des archives municipales ainsi que l'observation de réunions locales de concertation.

2 Pour Picon (2008), les scientifiques tentent de répondre à l'irruption de la question environnementale par le développement de l'interdisciplinarité entre sciences de la nature et sciences sociales.
}

d'un syndicat local chargé de l'entretien des digues communales et de la CNR, dont les aménagements traduisent une gestion extérieure et technicienne. À l'heure où la politique moderniste de grands travaux n'apparaît plus comme une réponse satisfaisante aux crues, comment se recomposent les relations entre la société locale et la puissance publique?

Enfin, l'expression «culture du risque » fait aujourd'hui florès, à l'instar de l'appel lancé par les responsables institutionnels pour une mobilisation des populations là où, auparavant, seule la puissance publique intervenait au nom d'une expertise technique. L'enquête conduite auprès des Barthelassiens montre comment les riverains vivent avec le risque au travers de pratiques enracinées dans l'expérience, mais aussi en étant confrontés à des enjeux qui dépassent le problème des inondations. Dès lors, le clivage entre experts et profanes reste-t-il pertinent, alors même que les savoirs locaux et la mémoire des catastrophes passées apparaissent aujourd'hui comme une ressource nouvelle pour les politiques de prévention ${ }^{3}$.

\section{Un territoire frontière : une île inondable entre ville et campagne}

La ville d'Avignon, qui fut pendant de longues années un État papal indépendant du royaume de France, était délimitée à l'ouest par une frontière fluctuante, qui correspondait au lit du Rhône, défini comme «l'intégralité des terres momentanément couvertes par les inondations", ainsi que ne manque pas de le rappeler la municipalité sur son site Internet, affirmant ainsi la présence du Rhône comme un élément fondateur de la cité. Le royaume de France s'étendait jusqu'au pied des remparts d'Avignon et, lorsque les crues inondaient les bas quartiers de la ville, les collecteurs royaux venaient en barque prélever les impôts dus à la couronne. Au cours de la Révolution française, la commune d'Avignon et le Comtat Venaissin furent rattachés à la République française par un vote de l'Assemblée nationale constituante. En 1793, le département du Vaucluse fut créé, avec pour chef-lieu Avignon. Toutefois, à cette époque, les îles de la Barthelasse et Piot appartenaient à la commune de Villeneuve-lès-Avignon, dans le département du Gard, car l'actuel bras mort du Rhône faisait alors frontière. Ce ne fut qu'en 1856, à la suite de l'intervention du conseiller général du Vaucluse, R. Lançon, auprès de l'Assemblée

\footnotetext{
3 À titre d'exemple, l'appel à projets lancé dans le cadre du volet inondation du Plan Rhône fait reposer la sensibilisation des populations au risque d'inondation sur la valorisation, à travers des démarches artistiques ou pédagogiques, d'un "savoir vivre avec le risque " présent au niveau local ( $c f$. http: //www.memoiredurisque08.fr).
} 


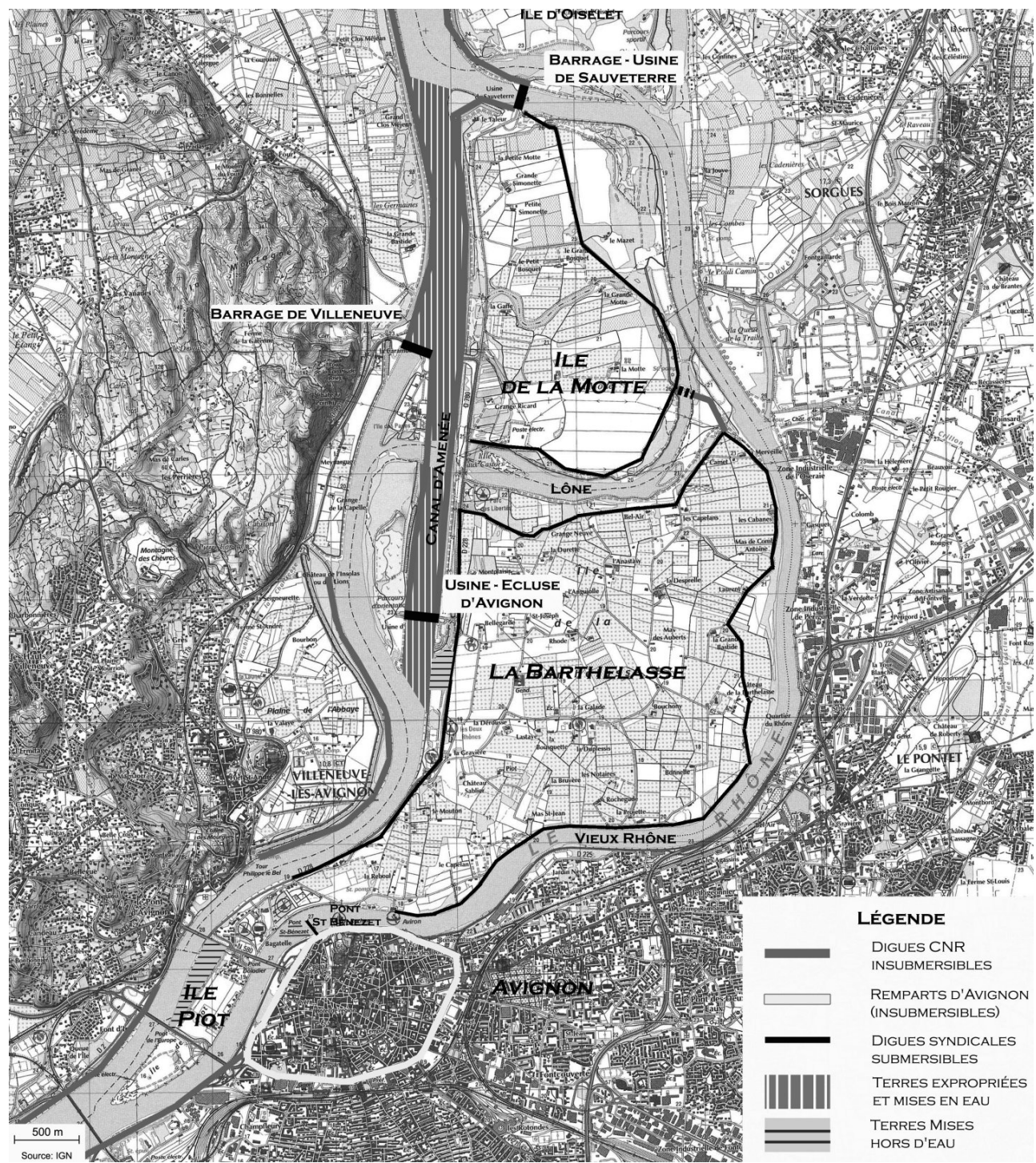

Figure : Situation de l'île de la Barthelasse.

nationale, que le rattachement de Piot et de la Barthelasse à Avignon sera prononcé ${ }^{\text {. }}$

Le découpage administratif de ce territoire suit donc les déplacements du fleuve à partir de frontières géographiques sans cesse redessinées par les crues. L'île de la Barthelasse est aujourd'hui devenue un quartier à part entière de la ville d'Avignon, qui y a implanté une mairie annexe, un camping municipal, une piscine, une école primaire et une maternelle, un centre de loisirs, un stade, une promenade aménagée, etc. La mise en place d'un réseau de bus et d'une navette fluviale pendant la saison estivale facilite enfin l'intégration de l'île dans l'agglomération.

Cependant, la Barthelasse apparaît comme une enclave et les habitants affirment leur identité insulaire en se reconnaissant plus volontiers Barthelassiens qu'Avignonnais. L'accès à l'île fut pendant longtemps impossible.

${ }^{4}$ Loi du 10 juillet 1856.
L'ancien pont d'Avignon fut emporté par le Rhône en 1669 , et le fleuve ne pouvait ensuite être traversé que par un bac, jusqu'à ce que le maire de la ville décide, en 1806, de construire un nouvel ouvrage en bois, puis un pont suspendu en 1843. L'isolement géographique est aujourd'hui interprété au regard des différences de modes de vie entre les ruraux barthelassiens et les citadins avignonnais.

L'agriculture bénéficie de la richesse agronomique des sols alluvionnaires, grâce aux inondations qui amènent du limon : "Vous avez peut-être trois mètres de limon, c'est des terres très fertiles. Ce sont des terres d'alluvions, alors, ce sont les meilleures terres que l'on pourrait avoir pour faire de l'agriculture... Avant, les terres d'alluvions avaient de superbes rapports. Maintenant, si on regarde bien ce que nous amènent les inondations comme pollution... » (une agricultrice). À l'heure des engrais et autres fertilisants, un agriculteur de la Barthelasse reconnaît que « le gain agronomique d'une inondation est 
insignifiant », mais, historiquement, la valeur culturale de l'île était élevée du fait de son caractère inondable ${ }^{5}$. La polyculture développée sur l'île reposait sur les céréales, qui nécessitaient de grandes surfaces et qui répondaient à la demande de la population de la ville toute proche, le maraîchage, à travers les jardins potagers des Avignonnais, et la viticulture, qui s'est développée à la fin du XIX ${ }^{\mathrm{e}}$ siècle. La submersion des vignes était alors le seul remède pour lutter contre le phylloxéra, qui ravageait de nombreux vignobles de Provence et du Languedoc. L'ouverture d'une cave coopérative ainsi que l'existence de nombreuses caves particulières témoignent de la prospérité assurée jusque dans les années 1970 par la production en quantité d'un vin de qualité médiocre. Suite à la crise viticole des années 1970, les jeunes agriculteurs se lancent dans l'arboriculture fruitière : abricotiers et pêchers, dont la rentabilité est meilleure que les fruits à pépins, pourtant moins vulnérables aux inondations.

Parallèlement à ces transformations agricoles, l'île s'est urbanisée jusqu'à former le quartier de Bagatelle, au sud de la Barthelasse et sur la partie nord de l'île Piot. Au regard de l'évolution de la tache urbaine de l'agglomération d'Avignon, l'urbanisation est restée très modeste sur ce territoire proche du centre-ville du fait des contraintes imposées par les documents réglementaires relatifs aux inondations du Rhône ${ }^{6}$. Un responsable des services municipaux explique ce mode d'urbanisation :

«Ce sont des gens qui, au départ, avaient des petits jardins, et ces petits jardins, parce que j'allais dire, c'est le paradis, l'île de la Barthelasse, par rapport à Avignon... Et petit à petit, si vous voulez, ils ont construit un cabanon, le samedi et le dimanche, ils venaient passer le week-end sur place, et après, ils ont obtenu des autorisations, des dérogations, on va dire, de la ville d'Avignon, à l'époque, pour construire... Et c'est pour ça qu'il y a aujourd'hui des secteurs urbanisés. Bon, au départ, c'était, ce n'était pas officiel, j'allais dire, $c^{\prime}$ était en principe "à la sauvage". Et petit à petit, ça s'est entériné. »

\footnotetext{
${ }^{5}$ L'île tirerait d'ailleurs son nom d'un certain Jean Richard, dit "Barthelucius », qui le premier, au XVe siècle, sema du blé sur la Barthelasse et fit fortune. Cette origine «légendaire », relatée par le site Internet de la ville d'Avignon (http://www.mairie-avignon. $\mathrm{fr} / \mathrm{fr} /$ pratique/transport/balade3.php), est controversée par certains habitants pour qui Barthelasse signifie « endroit humide».

${ }^{6}$ Au titre du libre écoulement des eaux et de la préservation des champs d'inondation, le plan des surfaces submersibles (PSS) réglemente à partir de 1982 les constructions dans le lit majeur du Rhône. Le plan d'occupation des sols (POS) de la ville d'Avignon, adopté en 1984 et révisé en 1996, classe les deux îles en zones ND, c'est-à-dire zones naturelles non urbanisables, à l'exception de certains droits de construction que conservent les agriculteurs. Depuis la crue de 1993, l'application de l'article R.111-3 du Code de l'urbanisme fige l'urbanisation sur le site. Enfin, le plan de prévention des risques inondations (PPRI), approuvé le 20 janvier 2000, place l'île de la Barthelasse en zone rouge, interdisant toute nouvelle construction, à l'exception des « hangars liés et nécessaires à l'exploitation agricole ».
}

Les restrictions à l'urbanisation ont permis de conserver la vocation agricole de l'île, en favorisant le regroupement des agriculteurs qui étaient les seuls à bénéficier de l'autorisation de construire jusque dans les années $2000^{7}$. En limitant l'installation de nouveaux habitants, cette réglementation a, de fait, renforcé l'interconnaissance des Barthelassiens, participé au développement de l'esprit « îlien » et contribué ainsi à la constitution d'une enclave agricole et insulaire jouant aujourd'hui le rôle de «poumon vert» pour la ville d'Avignon. Les familles sont souvent celles des agriculteurs installés sur l'île depuis trois ou quatre générations : «Il y a beaucoup de vieilles familles, oui... Et puis, il y a un esprit que l'on pourrait dire îlien » (ancienne institutrice de la Barthelasse).

L'affirmation de l'insularité renvoie aux rapports entre la ville et l'île fluviale et se cristallise autour de la solidarité territoriale mise en jeu par les inondations. Les digues de la Barthelasse ne protègent l'île que contre les faibles crues et permettent aux crues supérieures de déborder. Cet aménagement maintient l'inondabilité de la Barthelasse, ce qui diminue la pression sur la rive opposée et participe ainsi à la protection de la ville d'Avignon contre les inondations ${ }^{8}$. "On sait très bien que la protection de la Barthelasse ne peut se faire qu'au détriment de la protection de la ville d'Avignon, voilà. C'est-à-dire la protection de la partie urbanisée ne peut pas être remise en cause... C'est fondamental. On ne reviendra pas là-dessus » (un élu). Les habitants de la Barthelasse ne manquent pas de rappeler qu'ils sont inondés au profit des Avignonnais. «Toutes les villes sont plus ou moins protégées... Ce qui est logique... Mais au détriment des campagnes... Et ça, ça ne l'est plus trop, logique... » (une habitante). Un responsable du Syndicat des digues et des fossés explique de même : «On comprend que l'on veuille inonder des champs d'expansion ruraux... Oui, mais ça ne doit pas se faire à n'importe quel prix et pas n'importe quand. » Dans ce contexte, les Barthelassiens dénoncent, non pas l'inondabilité de l'île, ni même la protection de la ville à leurs dépens, mais l'absence de reconnaissance par les Avignonnais de leur «sacrifice» et de compensations par la puissance publique. La gestion des crues n'est pas sans conséquences dans les tensions déjà existantes

\footnotetext{
${ }^{7}$ Les recensements de l'INSEE indiquent une population résidente presque constante au cours des trente dernières années : 701 habitants en 1982, 934 en 1990 et 837 en 1999. On ne compte également que 12 résidences secondaires pour 377 logements en 1999 ( $c f$. http://www.insee.fr).

8 L'île de la Barthelasse est inondée à partir des crues de moyenne importance. L'écrêtement mesuré au profit de la ville d'Avignon est de 60 à $120 \mathrm{~m}^{3} / \mathrm{s}$ et le volume d'eau stockée de 11 à 43 millions de $\mathrm{m}^{3}$ suivant le type de la crue (Étude globale pour une stratégie de réduction des risques dus aux crues du Rhône : diagnostic hydraulique de la situation actuelle, Territoire Rhône / CNR, 2002, p. 25,). Aucune étude n'a cependant évalué l'impact précis qu'aurait sur Avignon la soustraction de l'île de la Barthelasse aux inondations du Rhône.
} 
entre les agriculteurs de la Barthelasse et les citadins d'Avignon : « Les Avignonnais se protègent... Donc, en se protégeant, eh bien, l'eau, elle arrive chez nous... [...] Et les Avignonnais, ils sont tous contents que l'eau vienne ici... C'est eux qui viennent prendre des photos pour nous voir dans l'eau! C'est agréable... C'est pour ça que d'un autre côté, non, le reste du temps, on n'est pas très contents quand on les voit arriver ici » (un habitant de l'île).

La solidarité territoriale face aux inondations est aussi questionnée sur le plan fiscal, car les habitants des îles Piot et de la Barthelasse acceptent mal de payer les mêmes impôts locaux que les habitants du centre-ville d'Avignon. Ils estiment être « les derniers des derniers », puisqu'ils ne bénéficient pas des services municipaux du centre-ville, alors qu'ils protègent celui-ci des crues du Rhône :

«Il n'y a pas d'éclairage, il n'y a pas de trottoirs. C'est des routes de campagne qui sont souvent boueuses, il n'y a pas de nettoyage... Alors qu'il y a des laveuses qui passent dans Avignon. On n'a jamais vu de laveuses... Donc, j'ai téléphoné à la mairie pour demander si c'était normal. Et la réponse a été très claire : "Mais oui, c'est normal... [...] Vous habitez dans un paradis, c'est normal que vous payiez!" Voilà la réponse de la mairie.»

La réponse de la municipalité justifie le taux élevé des impôts locaux par la qualité du cadre de vie de la Barthelasse, qui est apprécié selon les critères urbains et non pas comme un espace de travail agricole. La Barthelasse est considérée comme un "véritable paradis », une « campagne à cinq minutes de la ville » à laquelle rêvent les Avignonnais et non pas comme un île rurale inondable.

\section{Une gestion locale face à la norme de l'État aménageur}

Le fleuve est considéré après la Première Guerre mondiale au regard de son potentiel hydroélectrique. Alors que la destruction partielle du bassin houiller du Nord conduit à rechercher d'autres sources d'énergie, les chutes du haut Rhône sont l'objet d'un intérêt nouveau des industriels et du conseil municipal de Paris, qui pensent fournir ainsi le marché de la capitale. La Compagnie nationale du Rhône (CNR) est créée en 1933, sous la forme d'une société concessionnaire rassemblant les industries électriques, la Compagnie des chemins de fer de Paris à Lyon et à la Méditerranée (PLM), les collectivités rhodaniennes et le département de la Seine. Après la Seconde Guerre mondiale, les vagues de nationalisations modifient l'actionnariat de la CNR. La SNCF, EDF et les acteurs étatiques ont alors la majorité des voix au conseil d'administration et la CNR devient un acteur public incontournable de l'aménagement du territoire dans la vallée du Rhône.
Le principe du triple aménagement mis en œuvre le long du Rhône prévoit la réalisation de barrages hydroélectriques, mais aussi d'un chenal navigable et de réseaux d'irrigation pour l'agriculture. Alors que le «fleuve Dieu" ${ }^{9}$ » organise, au XIXe siècle, les activités humaines avec le développement de l'agriculture sur les sols limoneux, est mise en œuvre une "démarche de domestication de ce qui était jusqu'alors laissé au caprice de la nature et du temps (dans sa double acceptation météorologique et historique) » (Marié, 1996). L'idéal moderniste et techniciste guide jusqu'à la fin des années 1970 le triple aménagement du Rhône dans un contexte d'interventionnisme et de volontarisme étatique, pour aboutir à 18 aménagements hydroélectriques entre la Suisse et la Méditerranée, 330 kilomètres de voie navigable, la protection de 44000 hectares contre les crues et l'irrigation de 120000 hectares.

L'aménagement complexe de l'île de la Barthelasse, qui s'inscrit dans cette politique de grands travaux, débute en 1971 avec la construction de l'usine-barrage de Sauveterre, entre l'île de l'Oiselet et l'île de la Motte, le barrage de Villeneuve et le canal à l'intérieur de l'île qui amène l'eau jusqu'à l'usine-écluse d'Avignon. Le nouveau bras « vif » du Rhône est endigué sur toute sa longueur pour former le canal d'amenée, tandis que le bras «mort » d'Avignon, ou Vieux Rhône, permet les inondations de la Barthelasse. L'aménagement du canal usinier a nécessité des expropriations qui ont d'autant plus marqué les esprits que les terrains disparaissaient sous le Rhône, comme en témoignent les anciens habitants : «Dans les années 1960, 1965, ont eu lieu les premières expulsions... C'était violent, on a récupéré les terrains, abattu les maisons et fait passer le troisième bras du Rhône. » "Quand vous êtes en expropriation... c'est très dépressif ! C'est dur, parce que c'est la maison de vos ancêtres, la maison de vos parents, de vos grands-parents, qui disparaît pour des raisons... » La rectification du lit du Rhône conduit à une véritable "re-création ${ }^{10}$ " de la Barthelasse, dont l'inondabilité est bouleversée alors qu'elle était depuis longtemps l'objet d'une gestion locale. En temps normal, le tronçon court-circuité par la dérivation n'est plus alimenté que par un débit minimal garanti. En cas de crue, les barrages s'ouvrent progressivement et les débits se répartissent à nouveau entre les deux bras du Rhône. La Barthelasse est inondée par « remous aval ${ }^{11} »$, en face du pont Saint-Bénezet. Un habitant rend compte de ces bouleversements

\footnotetext{
9 Expression empruntée à Gilbert Tournier, 1952. Rhône, Dieu conquis, Paris, Plon.

10 Brochure de présentation de Donzère-Mondragon intitulée Le Nouveau Rhône est né, citée par Vernière (1999).

11 L'eau inonde la Barthelasse par l'aval de l'île et la remplit doucement. L'objectif est d'éviter un débordement par l'amont, qui créerait des courants traversants violents.
} 
qui effacent tout référent naturel :

«L'État, dans le cadre de l'aménagement du territoire, a supprimé des champs d'expansion et en a conservé d'autres. À partir de ce moment-là, il a changé la donne : on ne peut plus parler de champs d'expansion naturels, parce qu'il y en avait, des champs d'expansion naturels, et qu'il les a fermés. On a cherché à protéger les villes, donc on a soustrait des champs d'expansion. Il ne reste plus que $20 \%$ de plaines inondables, et ces $20 \%$ doivent assumer l'ensemble de l'écoulement du Rhône, ou de la gestion des inondations. Donc, on voit bien qu'il n'y a plus d'égalité. »

Pour les habitants, les aménagements apparaissent à l'époque «inexorables » et personne ne tente de les empêcher, car un des arguments avancés par la CNR serait la promesse d'une meilleure protection contre les crues : «Ils ont fait les barrages et ils nous ont dit : "Vous serez tranquilles, on va jeter des barrages et vous n'aurez plus d'inondations." Mais c'est l'inverse qui s'est produit...» «La CNR avait dit: "Vous n'aurez plus le Rhône!" Mais ce n'était pas vrai! Les vieux Barthelassiens leur ont dit, à la CNR: “C'est impossible de tenir l'eau qui arrive de là-haut pendant huit jours!" » Les aménagements de la CNR ont soustrait certains espaces aux inondations tout en pérennisant le rôle joué par des zones d'expansion de crue, comme la Barthelasse.

L'action de la CNR s'inscrit dans un objectif d'« aménagement » porté par une autorité centrale et technicienne, même si des formes de «ménagement ${ }^{12}$ » du territoire, de l'ordre de la négociation et du compromis, apparaissent dans les discours sur la meilleure protection contre les inondations ou sur l'apport de l'irrigation agricole. Elle rompt avec les modes de gestion assurés par une structure centenaire, enracinée dans le territoire, le Syndicat des digues et des fossés créé par l'ordonnance royale du 19 décembre 1841.

Même si le Syndicat des digues et des fossés a été imposé par le pouvoir royal, il repose sur les propriétaires qui doivent prendre en charge « la défense et [...] la conservation des bords de l'île de la Barthelasse » et participer, «dans la proportion de leur intérêt, aux dépenses des travaux à exécuter $»^{13}$. Après la crue de 1840 , de nombreux syndicats de propriétaires furent créés de manière forcée par l'État pour assumer financièrement les travaux de défense contre le Rhône ainsi que l'entretien des différents ouvrages de protection.

\footnotetext{
12 Expression empruntée à Brunet (1994), reprise par Marié (1996), qui explique à propos de l'aménagement du canal de Provence : «L'aménagement, comme processus volontaire d'organisation et de fertilisation de l'espace, ne réussit bien que s'il s'accompagne d'une certaine dose que l'on pourrait appeler le ménagement du territoire, que je définirais provisoirement comme étant la capacité des institutions de l'aménagement à autoréguler, c'est-à-dire à réévaluer en permanence les termes de leur action en fonction des forces en présence. »

13 Ordonnance royale de 1841.
}

Le programme général de défense contre les fleuves lancé par Napoléon III à la suite des inondations de $1856^{14}$ conduit à la construction de nombreuses digues hautes et discontinues qui s'abaissent vers l'aval pour laisser la crue envahir doucement les terres en remontant vers l'amont. À partir des années 1970, l'arrivée de nouveaux habitants conduit à des adaptations du système de financement, qui repose sur des liens forts entre le syndicat et le monde agricole. En 1974, une taxe est mise en place à la Barthelasse pour les habitations en plus de celle qui affecte les parcelles agricoles, de manière à ce que les résidents participent aux côtés des agriculteurs.

L'île de la Barthelasse est donc protégée par un système hybride constitué, d'une part, des digues CNR insubmersibles et, d'autre part, des digues syndicales, qui n'ont un effet que contre les petites crues. D'un côté, l'ambition moderniste est incarnée par l'État au travers de travaux prométhéens, de l'autre, la gestion locale repose sur les propriétaires fonciers, qui négocient la protection de l'île avec l'administration déconcentrée et, dans une moindre mesure, la municipalité. Alors que le syndicat n'a pas le droit de rehausser les digues à la hauteur des ouvrages de la CNR, car l'inondabilité de la Barthelasse doit être maintenue, une connaissance fine du terrain permet de ruser avec le « discours du code ${ }^{15}$ » :

«[...] parce que, entre le calcul mathématique et la réalité
physique d'une crue, c'est très différent. La végétation
fait un tel frein que vous avez des différences de niveau
considérables. Et ça n'est jamais pris en compte dans leurs
calculs. On ne dit rien, nous... Mais rien que le grillage, là,
c'est un barrage hydraulique de folie. Les feuilles se mettent
dans le grillage, et vous augmentez de $50 \mathrm{~cm}$ la protection...
Et ça, ils ne le savent pas, ils ne le voient même pas. Donc,
moi, je demande des choses que je sais acceptables et, à
la limite, je sais que par derrière je gagne... Je gagne 10 à
$15 \mathrm{~cm}$.» (Un responsable syndical.)

Pour obtenir une meilleure protection de la Barthelasse, les membres du syndicat recourent à un "grappillage » continu, centimètre après centimètre, en utilisant la végétation, voire le rehaussement des digues à l'occasion de travaux d'entretien. «Depuis plus de vingt ans, on a fait une politique de petits pas... Et on a truandé... On a truandé l'État. Quand ils nous disent: "Bon, OK, rebouchez les brèches...", eh bien, vous rebouchez les brèches, mais vous en mettez plus pour que ça tasse... Et si vous en mettez quand même beaucoup plus, vous gagnez

14 Annoncé dans la lettre de Plombières datée du 19 juillet 1856 : «Tout me fait espérer que la science parviendra à dompter la nature. Je tiens à l'honneur qu'en France les fleuves, comme la révolution, rentrent dans leur lit et qu'ils n'en puissent plus sortir. »

15 Pour Barel (1981), le code se définit comme « une mise en ordre de l'action et de la pensée prétendant s'abstraire de particularités spatiales et temporelles ", alors que le territoire, quant à lui, est «une force négatrice du code»: «le local commence là où le code s'arrête ». 
dix centimètres, alors voilà... » (un responsable syndical). Cette forme de résistance au règlement de la police des eaux est reconnue par les techniciens de l'administration : «On n'est pas dupes, on sait donc que ces digues fusibles vont casser, il va y avoir une reconstruction en urgence, ça veut dire qu'ils vont faire ce qu'ils voudront : les rehausser, les rendre moins cassantes, etc. Parce que, dans ces cas-là, on n'a aucun contrôle. » Cependant, les gestionnaires, tant du côté de l'administration que de la municipalité, tolèrent l'autonomie des habitants en échange de la responsabilité juridique des digues supportée par le syndicat : «Si c'est la ville qui est responsable des digues, ça ne se passera pas comme ça. Là, vous n'aurez rien à dire... Une fois que la ville prend sa décision au conseil municipal, c'est fini. Vous avez des avantages à rester maître du jeu, quand même... Alors ça a l'inconvénient de prendre du temps et vous êtes responsable, évidemment » (un responsable syndical).

Après les crues de 2002 et 2003, une association de riverains s'est constituée en complément du Syndicat des digues et des fossés. Le débat sur la protection de l'île de la Barthelasse entre agriculteurs et gestionnaires laisse la place aux revendications des habitants, qui se présentent sous la figure du sinistré pour réclamer des mesures compensatoires aux contraintes acceptées pour la protection d'Avignon. Les responsables institutionnels demandent, quant à eux, aux populations d'accepter de vivre avec les inondations et de développer une «culture du risque».

\section{Quelle «culture du risque » à la Barthelasse?}

La politique de prévention des risques connaît depuis peu une inflexion ${ }^{16}$ qui vise à limiter la construction d'ouvrages de protection et à développer la sensibilisation et l'éducation des populations, invitées à vivre avec les inondations. Au niveau national, le Conseil général des ponts et chaussées appelle à des visées éducatives : « Redonner à tous cette "culture générale" des manifestations relève de l'éducation et de l'action culturelle [...]. Ainsi, pourra évoluer dans un sens raisonnable le "terreau de fond" de l'opinion. Ainsi seront évitées des imprudences, bévues, des emballements de foule ${ }^{17}$. » Il s'agit d'inculquer aux profanes un mode d'appréhension technique des fleuves, des dangers et des risques associés. Au niveau local, le directeur départemental de l'Équipement du Vaucluse explique dans un forum d'acteurs : " Il faut que naisse aujourd'hui une réelle culture du risque chez tous, [car

\footnotetext{
${ }^{16}$ Cf. la loi du 30 juillet 2003 relative à la prévention des risques techniques et naturels et à la réparation des dommages.

17 Conseil général des ponts et chaussées, 2001. Risque naturels : prévention et communication, Paris, CGPC, p. 11.
}

c]e n'est pas dans la culture des gens ${ }^{18}$. Il s'agit de faire adopter aux populations des comportements rationnels en cas de crise et d'entretenir la "mémoire du risque ", qui doit participer à l'acceptation par la société locale des contraintes sur l'urbanisation. Le discours institutionnel de la culture du risque révèle la position surplombante des gestionnaires par rapport à une population peu «avertie » et peu « rationnelle». Cette approche prescriptive apparaît en décalage avec la relation construite dans le temps par les riverains avec le fleuve, relation indissociable de l'expérience des crues passées.

Les campagnes d'information consistent principalement à organiser des réunions publiques ou à distribuer de la documentation aux populations. Ces actions ne sont pas nouvelles, comme en témoigne la brochure publiée après la crue de 1935. Il s'agit alors d'expliquer aux Avignonnais «ce qu'ils devraient savoir » sur les crues et le système de protection municipale, de manière à permettre "aux générations futures de prophétiser sur les crues à venir ${ }^{19} »$. Ce document est en bien des points similaire aux plaquettes de sensibilisation distribuées aujourd'hui au nom de la culture du risque : photographies $\mathrm{du}$ territoire sous les eaux, chiffres pour rendre compte de l'importance des dégâts, chroniques des crues historiques... La réception de ces brochures est marquée par le scepticisme des habitants, pour qui le prospectus relève $\mathrm{d}$ 'une " communication sans rien derrière [...] uniquement pour de l'autosatisfaction ». Le message de prévention achoppe sur le procès fait à ces actions d'information qui ne serviraient qu'à dédouaner les gestionnaires de leurs responsabilités.

L'enquête menée auprès des habitants de la Barthelasse a révélé des modes complexes d'appréhension du risque d'inondation, qui, fondus dans le quotidien, ne sont pas réductibles à ces messages de prévention. Loin de l'image de populations urbaines désemparées face à la crue, les Barthelassiens ont souvent vécu plusieurs inondations et ont développé un savoir pratique qui leur permet une certaine anticipation : "On prévoit, quand c'est la saison, vers l'automne... un pack de charbon de bois, donc, on prévoit des bouteilles de gaz et un camping-gaz, pour se dépanner. » L'arrivée d'une crue donne lieu à de multiples préparatifs : voitures placées sur des points hauts, meubles montés à l'étage ou surélevés, constitution de réserves de nourriture, ou encore vérification de la disponibilité de la barque conservée par de nombreux agriculteurs. Les maisons comportent souvent au moins un étage et les terrains agricoles sont équipés de recati, sortes de buttes de terre surélevées pour sauver autrefois

\footnotetext{
18 Actes des forums d'acteurs du SCOT du bassin de vie d'Avignon, Aménager et développer face aux Risques, juillet 2005 : http://www.avignon-bassindevie.fr/rubrique.php? rubrique $\_$id $=44$ (Programme Forum 4, p. 18).

19 Anonyme, 1935. Inondations 1935 : Avignon et la Provence. Album documentaire, Avignon, Rullière Frères.
} 
le bétail et aujourd'hui maintenir le matériel hors de l'eau. Dans la plupart des fermes existaient aussi des encoules, plan inclinés pour faire monter à l'étage les animaux. Les observations réalisées par les habitants constituent, de plus, une expertise locale sans cesse renouvelée, comme l'explique un habitant de la Barthelasse :

«J'ai tout bien repéré les cotes... Dans mon atelier, en bas, toutes les cotes des niveaux. Eh bien, là, on est en conflit.. Ils disent : “La cote a été de 7,70 m"... Eh bien moi, je leur dis : "C'est pas vrai, la cote, au pont de l'Europe, nous avons eu 7,96 m, presque $8 \mathrm{~m}$." Et ça, je peux le prouver... Alors, l'autre jour, il y a l'ingénieur en chef, le directeur des services techniques de la ville... Et je lui ai dit: "Eh bien venez, passez en prendre, passez prendre des relevés, c'est tout marqué." »

En se mobilisant au travers d'associations, les riverains remettent en question le clivage entre experts et profanes et tentent de sortir du rôle d'élève, qui leur est imparti à travers les actions de "culture du risque ». Pour Bayet (2005), les associations de sinistrés modifient les rapports de force entre les acteurs : « Ils contraignent les pouvoirs publics à reconnaître que leurs besoins ne consistent pas seulement à retrouver une culture du risque. Ils montrent que leurs revendications ne découlent pas d'un calcul égoïste et d'une méconnaissance des phénomènes d'inondation, mais qu'elles s'inscrivent dans une relation au territoire, à son histoire et à ses institutions. » La montée en puissance de la parole riveraine interpelle des techniciens, qui reconnaissent à mots couverts que le savoir technique reste limité par une marge d'incertitudes : «Si on avait des bases techniques solides, des hypothèses solides, on pourrait dire effectivement : c'est là, et c'est pas autrement. Mais quand on connaît toutes les incertitudes au niveau du modèle, au niveau de la topographie, au niveau des hypothèses hydrauliques, météorologiques, des coefficients de rugosité que l'on met dans les modèles, je me dis, c'est un petit peu de la magie que l'on fait... » (un technicien de la DDE) ${ }^{20}$.

Enfin, les riverains ont constitué, avec l'aide de la mairie d'Avignon, une réserve communale de sécurité civile qui apporte un soutien aux équipes de secours, qui ne détiennent plus une connaissance fine du terrain depuis que les pompiers ne sont plus présents au niveau local. L'appel des gestionnaires à la culture du risque arrive en contradiction avec la perte d'interconnaissance entre les populations et les secours. Créée à la suite des dysfonctionnements de la crue de 2003, la réserve communale de sécurité civile rassemble une quinzaine d'habitants des îles, volontaires et bénévoles, qui renseignent les pompiers sur le territoire et la population. Ainsi, comme l'explique Vidal-Naquet (2001) : "Il est difficile de dire [...] que les populations riveraines sont dépourvues de

${ }^{20}$ Sur ce point, on pourra se reporter à l'article de Raccasi et al. (2008) sur les incertitudes inhérentes à la mise en place de modèles hydrauliques à partir de données anciennes sur des fleuves fortement aménagés, comme le Rhône ou le Danube. culture du risque, comme on le déplore souvent. Quand il n'est pas tenu à distance par l'autorité technique, le riverain aussi dûment "informé" qu'instruit par "l'expérience" prend nécessairement le risque en charge, ce sous la forme auto-organisée de pratiques individuelles, de stratégies de défense collectives, voire de valorisations identitaires. » L'expérience des crues constitue en effet un préalable pour être accepté comme Barthelassien par les « anciens ». La distinction entre ruraux et urbains est redessinée à l'aune de la différence entre ceux qui «savent ce que c'est » et ceux qui ne l'ont pas encore vécu :

«Mais là, vous avez énormément de maisons sur la Barthelasse qui ont changé de destinataires. Donc, aujourd'hui, on se trouve dans des situations qui sont beaucoup plus délicates, parce que les nouveaux propriétaires ne se rendent pas compte de ce que c'est qu'un Rhône. Tandis qu'avant, dès que les orages commençaient à tomber, vous alliez au Rhône, vous surveilliez... Ça reste dans la tradition du monde rural, ça. Mais pas dans celle des citadins qui habitent une zone rurale. Ils n'ont pas cette culture... C'est difficile de leur donner. Alors, ceux qui ont pris deux ou trois inondations, là, ils l'ont, ceux-là... Ils sont comme les agriculteurs, ils sont empreints de leur Barthelasse. » (Un responsable du Syndicat des digues et des fossés.)

À travers ce que les gestionnaires appellent «culture du risque ", s'établissent en pratique, pour les habitants, des distinctions sociales et des constructions identitaires qui font de l'expérience de l'inondation un ancrage au territoire et un élément d'appartenance à la société locale. Dans ce contexte, l'appel univoque et prescriptif des gestionnaires « serait le symptôme d'une véritable crise, [...] l'expression d'un désarroi des élites face à l'épaisseur sociale et la diversité des territoires » (Duchêne et MorelJournel, 2004).

\section{Conclusion}

La dialectique entre norme et territoire éclaire ce qui met l'île de la Barthelasse en tension entre la campagne et la ville, l'aménagement centralisé et la gestion locale, l'appel institutionnel à la culture du risque et les pratiques des populations. Ces trois clivages conduisent chaque fois à questionner l'équité territoriale qu'institue une gestion technique du fleuve et les principes de la politique de prévention des risques. La Barthelasse a historiquement participé à la protection d'Avignon contre les crues du Rhône et n'a pas bénéficié des aménagements de la CNR autant que d'autres territoires, soustraits tout ou partie aux inondations. Cette situation conduit aujourd'hui à des débats sur d'éventuelles compensations financières susceptibles d'être réclamées par ces territoires qui supportent des contraintes au profit d'autres espaces. La « scène locale du risque » (Decrop et al., 1997) reconstituée ici révèle ensuite la coexistence d'une gestion locale des 
agriculteurs, d'une logique d'aménagement globale portée par la CNR et d'une montée en puissance récente des riverains. Ces derniers sont invités par les gestionnaires à conserver la responsabilité de l'entretien des digues et à développer une «culture du risque ». Forts de ce rôle, les habitants demandent en retour plus de pouvoir de décision et contestent le monopole de l'expertise et le bien-fondé du contrôle réglementaire exercé par les responsables institutionnels. Le développement de pratiques et d'un savoir propres leur permet de s'affirmer comme acteurs et participe également à la construction d'une identité locale prise dans les tensions entre gestionnaires et administrés, entre urbains et ruraux.

\section{Références}

Barel, Y., 1981. Modernité, code, territoire, Annales de la recherche urbaine, 10-11, 3-21.

Bayet, C., 2005. Riverains inondables et défenseurs de l'environnement : mobilisations et contestations associatives dans le domaine de la prévention des inondations. Rapport pour le programme RI, CNRS-MEDD, Paris.

Reçu le 12 décembre 2008. Accepté le 2 avril 2009.
Brunet, R., 1994. La France, un territoire à ménager, Paris, Édition $\mathrm{n}^{\mathrm{o}} 1$.

Decrop, G., Dourlens, C., Vidal-Naquet, P.-A., 1997. Les Scènes locales de risque. Rapport de recherche, CERPE-Futur Antérieur, Lyon.

Duchêne, F., Morel-Journel, C. (Eds), Coanus, T., Martinais, E. (coll.), 2004. De la culture du risque : paroles riveraines à propos de deux cours d'eau périurbains, Paris, L'Aube.

Gentric, J., 2007. Inondation des villes, inondations des champs : l'interaction entre norme et territoire à partir des politiques de prévention des risques. Mémoire de master, ENTPE-IUL, Lyon.

Marié, M., 1996. Aménager ou ménager le territoire?, Annales des Ponts et Chaussées, 77, 67-76.

Picon, B., 2008. L'Espace et le temps en Camargue, $3^{\mathrm{e}}$ éd. revue et augmentée, Arles, Actes Sud.

Raccasi, G., Jugaru, L., Provansal, M., 2008. Incertitudes inhérentes à l'acquisition et au traitement de données anciennes dans la mise en place de modèles 2D, 3D et diachronique. Cas de deux milieux fluviaux : le bas Rhône et le delta du Danube, in Allard, P., Fox, D., Picon, B. (Eds), Incertitude et environnement, Aix-en-Provence, Edisud, 115-126.

Vernière, B., 1999. L'Action publique entre stabilité et changement : un siècle d'aménagement du Rhône, 1899-1999. Mémoire de DEA, IEP, Lyon.

Vidal-Naquet, P.-A., 2001. Inondations et culture du risque. Rapport de recherche, CERPE-Ministère de l'Équipement, Paris. 